\title{
On Optical CDMA MAC Protocols
}

\author{
Mohamed Aly A. Mohamed ${ }^{1}$, Student Member, IEEE, Hossam M. H. Shalaby, Senior Member, IEEE, and \\ El-Sayed A. El-Badawy, Senior Member, IEEE \\ Dept of Elect. Eng., Faculty of Eng., Univ. of Alexandria, Alexandria 21544, Egypt \\ ${ }^{1}$ Also, Huawei Tech Co., Network Application and Software Dept, Egypt Representative Office, Cairo, Egypt.
}

$\left\{m \_a l y\right.$, shalaby,sbadawy\}@ieee.org

\begin{abstract}
A media access control (MAC) protocol for optical code-division multiple-access (CDMA) packet networks with variable length data traffic is proposed. This protocol exhibits a sliding window with variable size. A model for interference level fluctuation and an accurate analysis for channel usage is presented. Both multiple access interference (MAI) and photodetector's shot noise are considered. Both chip-level and correlation receivers are adopted. The system performance is evaluated using traditional average system throughput and average delay. Finally, we apply error control codes (ECCs) in order to enhance the overall performance. Our results indicate that the performance can be enhanced to reach its peak using ECC with optimum number of correctable errors. Furthermore chip-level receivers are shown to give much higher performance than that of correlation receivers. Also, it has been shown that MAI is the main source of signal degradation.
\end{abstract}

\section{INTRODUCTION}

Optical fibers have been commonly used in many communications and computer networks due to its extremely high bandwidth and its very low power losses. Our aim in this paper is to analyze an unslotted optical CDMA packet network and measure its performance. Each terminal in the network breaks its variable-length message into a set of constant length packets. The message length is variable. Two main indicators of the system performance have been focused upon. The first is the network throughput in packets per slot (packet duration). The second indicator is the network delay. Upon successful reception of packets, the receiver sends positive acknowledgment to the transmitter. Packet failure, however, is detected due to lack of positive acknowledgement.

The rest of the paper is arranged as follows. In Section II the network architecture is presented. The mathematical model of the system is illustrated in Section III. In Section IV the packet success probability, system throughput, and system delay are evaluated. Our numerical results are shown in Section V. Finally the paper is concluded in Section VI.

\section{NETWORK ARCHITECTURE}

The proposed network consists of a large number of users that can be considered as infinite population network. The network topology is a centralized network (star topology). Spread spectrum multiple access technique is applied with a common spreading sequence and the receiver can distinguish between time overlapped packets if there is a time offset that is greater than the width of the auto-correlation function of the used spreading sequence. In unslotted systems we can assume that the receiver can distinguish between all received packets. At the physical layer we consider both chip-level and correlation receivers.

The traffic offered to the system is assumed to be Poisson with average rate of $\lambda$ messages $/ \mathrm{sec}$. Each packet consists of a fixed number of bits ( $K$ bits/packet) and the message length $B$ (packets/message) is geometrically distributed with average length of $B_{a v}$ and a maximum length of $B_{\max }$; then the message length is $B \times K$. An error control code is applied and can correct up to $t$ errors/packet. The near-far effect is neglect and all packets arrive to the receiver with equal power.

The system uses OOK modulation scheme and applies a spreading sequence from optical orthogonal code (OOC) family ( $N, w, \lambda_{a}, \lambda_{c}$ ), where $N$ is the code length, $w$ is the code weight, and $\lambda_{a}, \lambda_{c}$ are the maximum values of the auto- and cross-correlation functions, respectively [1]-[3]. Both $\lambda_{a}$ and $\lambda_{c}$ are bounded to one. The average bit error probability for the correlation receiver under the influence of MAI only is [6]:

$P_{b}(m)=\frac{1}{2} \cdot \sum_{i=w}^{m}\left(\begin{array}{l}m \\ i\end{array}\right) \cdot\left(\frac{w^{2}}{2 N}\right)^{i} \cdot\left(1-\frac{w^{2}}{2 N}\right)^{m-i}$

where $m$ is the number of interferers and $K$ is the packet length.

As for the chip level receiver the bit error probability with shot noise and MAI is written as follows [4]:

$P_{b}(m)=\frac{1}{2} \cdot\left[1+\sum_{i=1}^{w}(-1)^{t} \cdot\left({ }_{i}^{w}\right) \cdot\left(1-e^{-Q i}\right) \cdot\left[1-i \cdot \frac{w}{2 \cdot N}+i \cdot \frac{w}{2 \cdot N} \cdot e^{-Q}\right]^{m}\right],(2)$

where $Q$ is the average photons per a chip pulse. When $Q-\propto$ the bit error probability reduces to [4]

$$
P_{b}(m)=\frac{1}{2} \cdot\left[1+\sum_{i=1}^{p}(-1)^{i} \cdot\left(\begin{array}{l}
w \\
i
\end{array}\right) \cdot\left[1-i \cdot \frac{w}{2 \cdot N}\right]^{m}\right] .
$$




\section{System Analysis And Mathematical Model}

In this section it we illustrate the mathematical model in order to evaluate both the system throughput and delay versus the system offered traffic. First we evaluate the traffic offered to the system, then the transition of the interference level, and finally the packet success probability.

\section{A. Average Offered Traffic (G)}

The system average offered traffic is defined as the average number of generated packets per packet duration:

$$
G=\lambda \cdot T_{p} \cdot B_{a v},
$$

where $T_{p}$ is the packet duration. Since the length of a message is assumed to be geometrically distributed, the probability of a message to be of length $x$ is given by

$$
P_{B}(x)=\alpha \cdot p \cdot(1-p)^{x-1},
$$

where: $\quad x \in\left\{1,2, \ldots, B_{\max }\right\}, \quad p=1 / B_{a v}$, and $\alpha=\frac{1}{1-(1-p)^{B_{\max }}}$ (6)

\section{Modeling of the Interference Level and Transition}

The interference level of the unslotted systems is characterized by continuous change, Fig. 1, shows the fluctuation of the interference level. The generation of new messages is assumed to follow the Poisson distribution with arrival rate ( $\lambda$ messages $/ \mathrm{sec}$ ). While, the termination of transmitted messages. Then, the prob. of generating $k$ new messages is:

$$
P_{o}(k, t)=\frac{(\lambda \cdot t)^{k}}{k !} \cdot e^{-\lambda t} .
$$

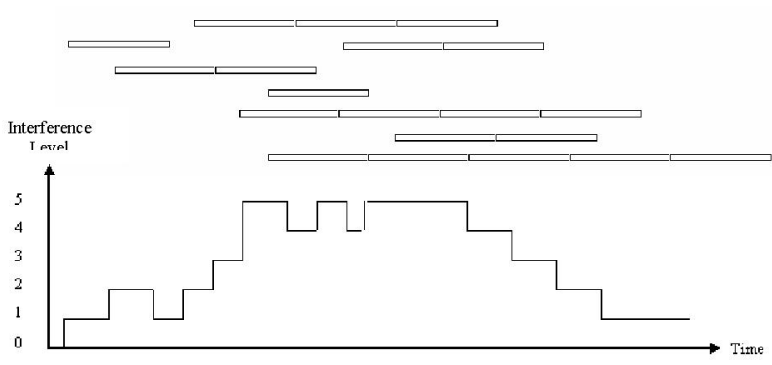

Figure 1. Interference level fluctuation

We found that for a data rate $R=256 \mathrm{kbps}$, average message length $B_{a v}=2$, average offered traffic $G=5$ packets/slot, and a packet length $K=128$ bits, the probability of generating $k$ - where $k=0,1,2,3,4,5$ - messages in a bit duration is $\left\{0.98,0.019,1.8 \cdot 10^{-4}, 1.2 \cdot 10^{-6}, 5.9 \cdot 10^{-9}\right.$, $2.3 \cdot 10^{-11}$ \}. Thus, we will neglect the probability of generating more than one bit in a bit duration, and the transition of the interference level is limited to one.

As for the termination process it is quite difficult to evaluate its rate, however, this process is also expected to follow the Poisson distribution. In the next section we will evaluate the average death rate $\mu\left(m_{1}\right)$ in terms of the number of interferes at the beginning of the tagged packet.

Next, we evaluate the interference level during the tagged packet. We assume Markovian model in our analysis, Fig. 2.

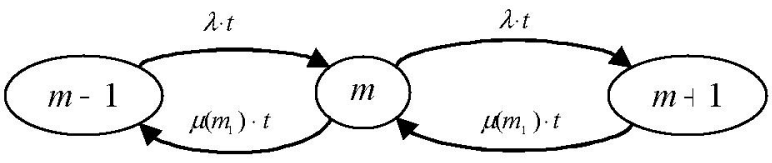

Figure 2. Transition of the interference level

Since the interference level can be changed by a value of 1 during a bit, and assuming that the level is $m$ at a certain bit, the level of the next bit may be $m \dashv 1, m-1$, or $m$.

Thus, the transition probability can be calculated as follows:

$$
q\left(m_{j} \mid m_{j-1}\right)=\left\{\begin{array}{lc}
\lambda \cdot T_{b} & m_{j}=m_{i-1}+1 \\
\mu\left(m_{1}\right) \cdot T_{b} & m_{j}=m_{i-1}-1 \\
1-\lambda \cdot T_{b}-\mu\left(m_{1}\right) \cdot T_{b} & m_{j}=m_{i-1} \\
0 & \text { otherwise }
\end{array}\right.
$$

where $\mu\left(m_{1}\right)$ is the death rate, which is the rate of message's termination. Now, it is required to express the average death rate, this is to be discussed in the following steps.

As shown in Fig. 3, the tagged packet method is used to analyze the system. The time axis is divided into periods each of length $T_{P}$, the tagged packet occupies the period number $\tau$. Let $\tau_{\mathrm{j}}$ denote the $j^{\text {th }}$ bit in the period $\tau$. In the tagged packet, $m_{j}$ is the interference level at $j^{\text {th }}$ bit. The interference level during the tagged packet is changed continuously; for simplicity it will be considered constant during each bit and changes occur at the bit boundaries. In order to study the interference level during the tagged packet we should consider both the generation and termination (death) rate. The generation rate is assumed to be a Poisson process.

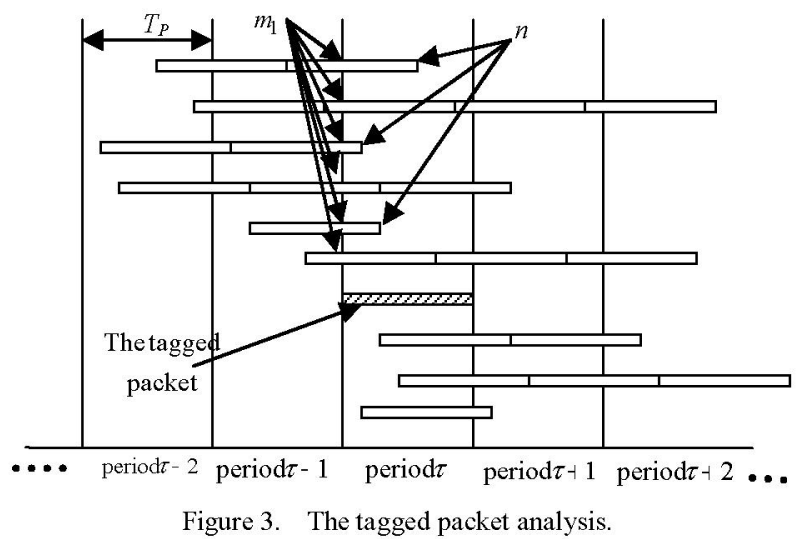

Figure 3 . The tagged packet analysis. 
It is now required to calculate $P_{I}\left(m_{1}\right)$, the probability of initial interference level to be $m_{1}$. Define: $a_{y}$ : number of interferers existing at $\tau_{1}$ that have been generated in the period $\tau-\mathrm{y} ; 1 \leq \mathrm{y} \leq B_{\max }$. And $A=\left\{a_{1}, a_{2}, \ldots, a_{y}, \ldots\right\}:$ set of all interferers generated in the previous $B_{\max }$ periods and exists at $\tau_{1}$.

Now, consider that $k$ messages are generated in the period $\tau-\mathrm{y}$ and any arbitrary $a_{y}$ messages among $k$ have a sufficient length to survive at the beginning of the tagged packet. Thus, $\operatorname{Pr}\left(a_{y}\right)$ follows the binomial distribution as follows:

$\operatorname{Pr}\left(a_{y}\right)=\sum_{k=a_{y}}^{\infty}\left(\begin{array}{l}k \\ a_{y}\end{array}\right) \cdot\left(P_{B}(B \geq y)^{a_{y}} \cdot\left(1-P_{B}(B \geq y)^{k-a_{y}} \cdot P_{o}\left(k, T_{P}\right)\right.\right.$

where. $P_{B}(B \geq y)=\sum_{x=y}^{B_{\max }} P_{x}(x)$

Then the probability to have a set of interferences $A$ : $\left\{a_{1}, a_{2}, \ldots . a_{y} \ldots\right\}$ can be obtained by multiplying the probabilities of $a_{y}{ }^{\prime} s$ as: $\operatorname{Pr}(A)=\prod_{y=1}^{B \max } \operatorname{Pr}\left(a_{y}\right)$.

Now, it is easy to calculate the probability of having $m$ initial interferences; it is the sum of the probabilities of all $A$ 's whose summation is $m$ as:

$$
P_{I}(m)=\sum_{u} \operatorname{Pr}(A),
$$

where $u=\left\{A: \forall a_{y} \in A ; \sum_{y=1}^{B_{\operatorname{mag}}} a_{y}=m\right\}$.

In this model it is assumed that the packet termination is a Poisson process with average rate of $\mu(m)$ packets/sec. In order to evaluate the average death rate $\mu(m)$, suppose that the number of initial interferers in the tagged packet is equal to $m$, and that $n$ messages among $m$ will depart during the tagged packet. A message will depart during the tagged packet if it was initiated in the period $\tau$ - $y$ and its length is equal to $y$. Define the probability $P_{d 1}(m)$ as follows: the probability of one message to be of length $y$ and generated in the period $\tau-y$.

$$
P_{d 1}\left(m_{1}\right)=\sum_{y=1}^{B_{\text {max }}} \sum_{a_{y}=1}^{m_{1}} \operatorname{Pr}\left(a_{y}\right) \cdot a_{y} \cdot P_{x}(y) \cdot\left(1-P_{B}(y)\right)^{a_{y}-1} .
$$

Therefore, for a given value of initial interference $m_{1}$, the probability of $n$ messages to be terminated in the tagged period follows the binomial distribution as shown:

$$
\operatorname{Pr}\left(n \mid m_{1}\right)=\left(\begin{array}{l}
m_{1} \\
n
\end{array}\right) \cdot\left(P_{d 1}\left(m_{1}\right)\right)^{n} \cdot\left(1-P_{d 1}\left(m_{1}\right)\right)^{m_{1}-n} .
$$

The average value of $n$, for a given value of $m$, is :

$$
n_{a v}\left(m_{1}\right)=\sum_{n=1}^{m_{1}} n \cdot \operatorname{Pr}\left(n \mid m_{1}\right)
$$

Finally, we can express the average death rate, for a given value of initial interference $m_{1}$, as follows:

$$
\mu\left(m_{1}\right)=\frac{n_{a v}\left(m_{1}\right)}{T_{P}} .
$$

\section{System PERFormance}

In this section it is required to evaluate recursively the number of errors in the packet. The packet will be successfully transmitted if the number of errors is less than or equal to $t$, the number of correctable bits by ECC. Define an error counter, $f_{j}\left(e, m_{j}, m_{1}\right)$, such that In the tagged packet the first $j$ - 1 bits are transmitted with $e$ errors. Such that the initial condition for the recursive calculations as follows:

$$
f_{1}\left(e=0, m_{1}, m_{1}\right)=P_{I}\left(m_{1}\right), \quad f_{1}\left(e>0, m_{1}, m_{1}\right)=0
$$

Considering the Markovian property of the interference level transition, the $f_{j}$ function can be expressed as follows:

$$
\begin{gathered}
f_{j}\left(e, m_{j}, m_{1}\right)=\sum_{m_{j-1}=m_{j}-1}^{m_{j}+1} f_{j-1}\left(e-1, m_{j}, m_{1}\right) \cdot q\left(m_{j} \mid m_{j-1}\right) \cdot P_{b}\left(m_{j-1}\right) \\
\quad+\sum_{m_{j-1}=m_{j}-1}^{m_{j+1}} f_{j-1}\left(e, m_{j}, m_{1}\right) \cdot q\left(m_{j} \mid m_{j-1}\right) \cdot\left(1-P_{b}\left(m_{j-1}\right)\right)
\end{gathered}
$$

Finally, the packet success probability is the probability that the number of errors does not exceed $t$, the maximum correctable errors by Reed-Solomon (RS) codes, at the last bit of the tagged packet using the $f_{j}$ function till $j=K$

and averaging over all possible values of $m_{K}$ and $m_{1}$. The packet success probability $Q_{s}(t)$ is expressed as follows:

$$
Q s(t)=\sum_{m_{K=0} m_{1}=0}^{\infty} f_{K}^{\infty}\left(t, m_{K}, m_{1}\right) \cdot\left(1-P_{b}\left(m_{k}\right)\right)+\sum_{e=0}^{t-1} f_{K}\left(e, m_{K}, m_{1}\right)
$$

The packet success probability $(Q S)$ is given by (19) and the system throughput is given by:

$$
S=G \cdot Q s(t) \cdot \frac{s}{r}=G \cdot Q s(t) \cdot\left(1-\frac{2 \cdot t}{K}\right)
$$

The last factor in (20) considers the effect of bandwidth expansion by the RS coding; where $s$ is the number of data bits/code word of length $r$, and the number of correctable bits is given by $t=(r-s) / 2$. In this system the packet is considered as one code word i.e $r=L$. Then, the average delay is: $D_{t}=G / S_{t}$.

\section{NUMERICAL EXAMPLES}

In this section we will present some numerical results and examine the performance of the network. The parameters used in analysis are: bit rate of $256 \mathrm{Kbps}$, average message length of 2 packets per message, and maximum message length of 3 packets per message. The used OOC is of length 127 and of weight 3 . 
Figure 4 compares the performance of correlation and chip-level receivers. It is clear that the system throughput achieved by the chip-level receiver is much higher than that of the correlation receiver.

Figure 5 examines the effect of ECC with different values of $t$. The use of ECC is expected to enhance the system performance. We consider two factors; the first is the enhancement obtained in the packet success probability; this enhancement is proportional to the number of correctable bits. The second is the bandwidth expansion due to the addition of parity bits. As shown in Fig. 4 we can see that the highest performance is achieved at $t$ is 3 .

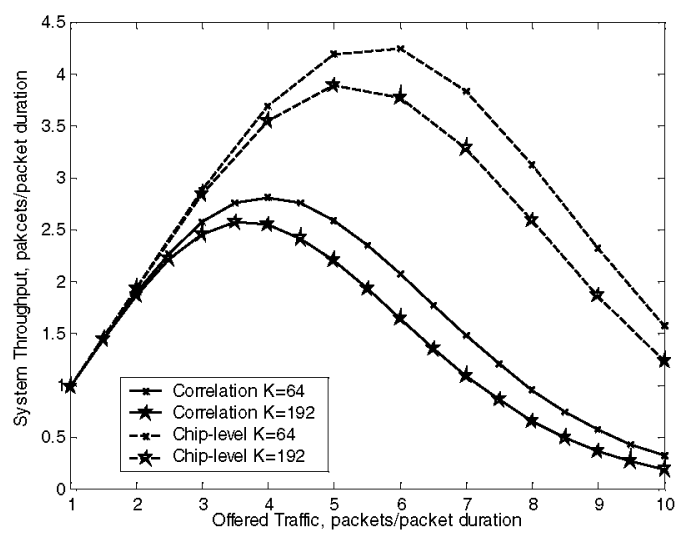

Figure 4. System throughput versus offered traffic for both correlation and chip-level receivers.

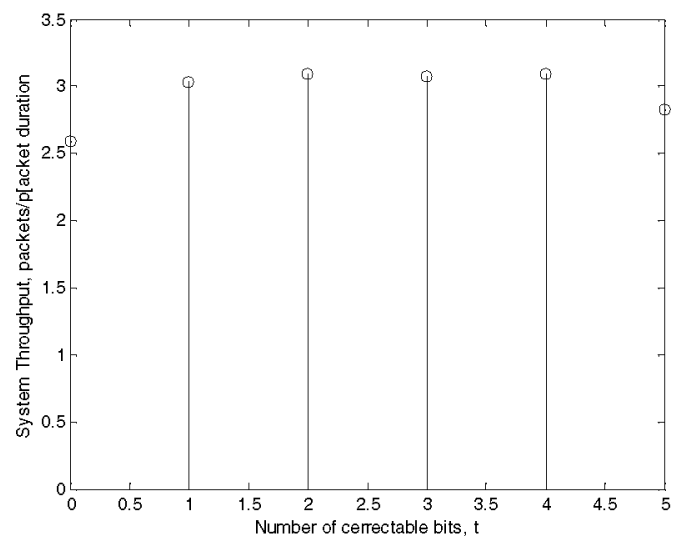

Figure 5. System throughput versus number of correctable bits $t$ for the correlation receiver, $G=5, K=64$.

Finally, Fig. 6 presents the effect of the photo detector's shot noise on the performance of chip-level receiver. It is found that the system throughput reaches its maximum when $Q$ exceeds about 10 photons per chip pulse. Hence, we can conclude that MAI is the major source of error

\section{CONCLUSION}

An accurate analysis of MAC protocol used in optical CDMA networks is presented. Sliding window protocol with variable size is considered. The system throughput and average delay are evaluated and an accurate description of the system state and channel usage is provided. Furthermore, the achieved enhancement by error control codes (ECCs) is examined. System performance is examined under the influence of both MAI and photodetector's shot noise. Results show the average system throughput and average delay versus the offered traffic, and average number of photons per chip pulse, as well as the effect of using ECC. Results indicate that the system performance is enhanced with the increment of number of correctable bits $t$ reaching a maximum value. Moreover, as $t$ increases the throughput decreases. It is also concluded that a significant improvement in the performance can be achieved using chip-level receiver instead of the traditional correlation receiver. As for the effect of photodetector's shot noise, we have found that it has a minor effect on the performance compared to MAI.

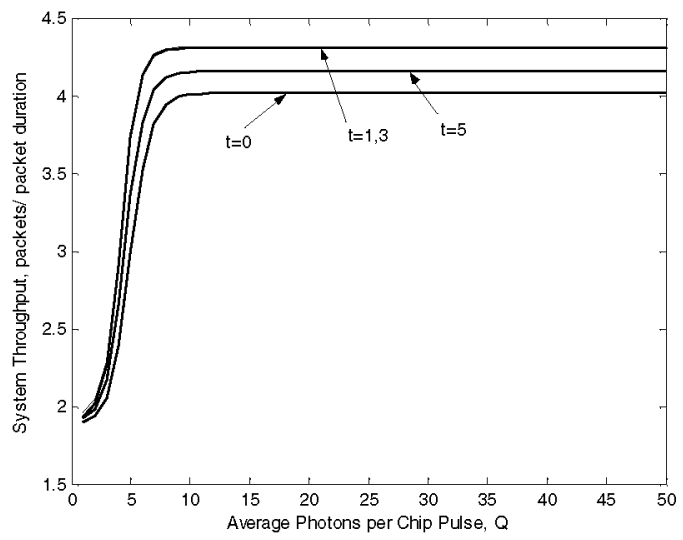

Figure 6. System throughput versus average number of photons per chip pulse for chip-level receiver, $G=5, K=64$.

\section{REFERENCES}

[1] J. A. Salehi, "Code division multiple-access techniques in optical fiber networks-Part I: Fundamental principles," IEEE Trans. Commun., vol. 37, pp. 824-833, Aug. 1989.

[2] J. A. Salehi, "Code division multiple-access techniques in optical fiber networks-Part II: Systems performance analysis," IEEE Trans. Commun., vol. 37, pp. 834-842, Aug. 1989.

[3] F. R. Chung, J. A. Salehi, and V. K. Wei, "Optical orthogonal codes: Design, analysis, and applications," IEEE Trans. Inform. Theory, vol 35, pp 595-604, May 1989.

[4] H. M. H. Shalaby, "Chip-level detection in optical code-division multiple-access," IEEE/OSA J. Lightwave Technol., vol. 16, pp. 10771087, June1998.

[5] D. H. Davis and S. A. Gronemeyer, "Performance of slotted ALOHA random access with delay capture and randomized time of arrival," IEEE Trans. Commun., vol. COM-28, No. 5, pp. 703-710, May. 1980.

[6] J. Muchenheim, and D. Hampicke, "Protocols for optical CDMA local area networks" Proc. NOC'97, vol. 1, (Antwerpen), pp. $255-$ 262, 1997

[7] J. So, Il Han, B. Shin and D. Cho, "Performance analysis of DS/SSMA unslotted ALOHA with variable length data traffic," IEEE J. Select. Areas Commun., vol. 19, No. 11, Nov. 2001.

[8] M. A. A. Mohamed, H. M. H. Shalaby, and El-Sayed A. El-Badawy "Variable-size sliding window optical CDMA MAC protocol," The $46^{\text {th }}$ IEEE International Midwest Symposium on Circuits and Systems (MWSCAS'2003), December 27-30, 2003, Cairo, Egypt. 